\title{
DESEMPEÑO AGRONÓMICO DE GENOTIPOS DE Brachiaria humidicola (Rendle) Schweickt EN EL TRÓPICO HÚMEDO DE MÉXICO
}

\author{
AGRONOMIC PERFORMANCE OF Brachiaria humidicola (Rendle) Schweickt \\ GENOTYPES IN THE MEXICAN HUMID TROPICS
}

\author{
Pedro I. Cruz López ${ }^{1}$, Alfonso Hernández Garay ${ }^{1 \star}$, Javier F. Enríquez Quiroz ${ }^{2}$, Sergio I. Mendoza Pedroza ${ }^{1}$, \\ Adrián R. Quero Carrillo ${ }^{1}$ y Bertín M. Joaquín Torres ${ }^{3}$
}

\footnotetext{
${ }^{1}$ Posgrado en Recursos Genéticos y Productividad-Ganadería, Colegio de Postgraduados-Campus Montecillo. 56230, Montecillo, Texcoco, Estado de México ${ }^{2}$ Sitio Experimental Papaloapan, Instituto Nacional de investigaciones Forestales Agrícolas y Pecuarias. Isla, Veracruz, México. ${ }^{3}$ Universidad del Papaloapan. Loma Bonita, Oaxaca.
}

*Autor para correspondencia (hernan@colpos.mx)

\section{RESUMEN}

En la región tropical de México, donde la producción bovina se basa en sistemas de pastoreo extensivos, los pastos del género Brachiaria son de importancia debido a su alto rango de adaptación, buen rendimiento y calidad nutricional. El objetivo del presente estudio fue evaluar el rendimiento total anual, composición morfológica y tasa de crecimiento de 23 genotipos de Brachiaria humidicola, más un híbrido de Brachiaria. El estudio se efectuó de junio 2006 a mayo de 2007 en Isla, Veracruz, México. Los tratamientos fueron evaluados cada $28 \mathrm{~d}$ en la época de lluvias y cada $42 \mathrm{~d}$ en las épocas de nortes y de seca, y distribuidos aleatoriamente en un diseño de bloques al azar con tres repeticiones. En rendimiento total hubo diferencias entre genotipos ( $\mathrm{P} \leq \mathbf{0 . 0 5})$, con el genotipo CIAT $679 \mathrm{cv}$. 'Chetumal' (17 $353 \mathrm{~kg}$ $\mathrm{MS} \mathrm{ha}^{-1}$ ) con la mayor producción, y el genotipo CIAT 26159 el de menor rendimiento (11 $104 \mathrm{~kg} \mathrm{MS} \mathrm{ha}^{-1}$ ). La distribución estacional del rendimiento total, en promedio de los 24 genotipos, fue: $83 \%$ en lluvias, $9 \%$ en nortes y 8 \% en sequía. La mayor tasa de crecimiento se presentó en el mes de junio con un promedio de $189 \mathrm{~kg} \mathrm{MS} \mathrm{ha}^{-1} \mathrm{~d}^{-1}$, que se redujo gradualmente hasta $4 \mathrm{~kg}$ MS ha-1 $\mathrm{d}^{-1}$ en mayo. El componente con mayor contribución al rendimiento fue el de hojas, con la siguiente tendencia estacional: nortes $>$ seca $>$ lluvias $(\mathrm{P} \leq \mathbf{0 . 0 5})$, con promedios de $77.6,71.7$ y $57.6 \%$, respectivamente.

Palabras clave: Brachiaria sp., rendimiento de forraje, tasa de crecimiento.

\section{SUMMARY}

In the Mexican tropical region, where animal production is based on extensive grazing systems, the grasses of Brachiaria genus are important due to their good adaptation, high forage yield and nutritive value. The aim of this study was to evaluate forage yield, morfological composition and growth rate of 23 genotypes of Brachiaria humidicola plus one hybrid of Brachiaria. The study was carried out from June 2006 to May 2007, at Isla, Veracruz, México. Treatments were measured every $28 \mathrm{~d}$ during the rainy season and every $42 \mathrm{~d}$ during the dry and northern cold-front seasons, and distributed in a completely randomized block design with three replicates. There were significant differences in total forage yield $(P \leq 0.05)$, with genotype CIAT $679 \mathrm{cv}$. 'Chetumal' being the highest with $17353 \mathrm{DM} \mathrm{kg} \mathrm{ha}^{-1}$ and CIAT 26159 the lowest with $11104 \mathrm{~kg} \mathrm{DM} \mathrm{ha}^{-1}$. The average seasonal forage yield distribution of the 24 genotypes was 83,9 and $8 \%$ during the rainy, northern cold-front and dry seasons, respectively. The highest average growth rate was recorded in June with $189 \mathrm{~kg} \mathrm{DM} \mathrm{ha}^{-1} \mathrm{~d}^{-1}$, which gradually decreased to $4 \mathrm{~kg}$ $\mathrm{DM} \mathrm{ha}^{-1} \mathrm{~d}^{-1}$ in May. Leaves contributed most to forage yield, and the seasonal trend was northern cold-front $>$ dry $>$ rainy seasons, with averages of 77.6 , 71.7 y $57.6 \%$, respectively.

Index words: Brachiaria sp. forage yield, growth rate.

\section{INTRODUCCIÓN}

En la ganadería que se practica en el trópico de México, los pastos son la base de la alimentación animal. En esta región hay diversos factores que limitan la producción y persistencia de los forrajes, como son: suelos con $\mathrm{pH}$ ácido, niveles altos de aluminio y manganeso intercambiables, baja disponibilidad de nutrientes y de materia orgánica y deficiente manejo de las praderas, factores que en conjunto provocan baja persistencia en la mayoría de las especies forrajeras introducidas. En la zona tropical, los pastos introducidos como 'Guinea' (Panicum maximun Jacq.), 'Pangola' (Digitaria decumbens Stent.) y 'Estrella africana' (Cynodon nlenfuensis Vanderyst) están siendo sustituidas por gramíneas de introducción reciente, como las variedades 'Mombasa' y 'Tanzania' (Panicum máximum Jacq.), así como variedades del género Brachiaria como 'Senal' (B. decumbens Stapf.), 'Insurgente' (B. brizantha (Mochst) Stapf.) y 'Humidícola' (B. humidicola (Rendle) Schweickt) (Enríquez, 1999).

Los pastos del género Brachiaria ofrecen nuevas expectativas para la ganadería tropical de Centro y Sudamérica (Keller-Grein et al., 1996). En Brasil hay 197 millones de hectáreas de pastizales de las cuales más de $60 \%$ están cubierta por especies cultivadas en las que predominan especies del género Brachiaria (FAO, 2006). En México las especies de Brachiaria se han expandido rápidamente y se 
estima que representan $6.5 \%$ del área cubierta por pastos (2.6 millones de hectáreas) (Argel, 2005).

Las variedades 'Insurgente’ y 'Chontalpo' están ampliamente difundidas por su amplio rango de adaptación, buen rendimiento y aceptable valor nutritivo (Keller-Grein et al., 1996). En particular, B. humidicola presenta amplio potencial para el trópico húmedo mexicano por su buena adaptabilidad a suelos anegados o inundados, soporta alta carga animal debido a su hábito de crecimiento estolonífero, tolera el ataque de la mosca pinta (Aeneolamia postica) y se adapta fácilmente a suelos ácidos de baja fertilidad (Enríquez, 1999).

Sin embargo, la variación de las condiciones climáticas a través del año interfiere en la estacionalidad de la producción, productividad y la calidad del forraje (Cruz y Boval, 2000). Así, en la región costera del Golfo de México el crecimiento de las plantas es determinado por tres épocas en el año: 1) Época de lluvias, de junio a octubre, cuando la precipitación y temperatura favorecen el crecimiento de las plantas; 2) Época de nortes, de noviembre a febrero, en la que disminuye el crecimiento de la mayoría de las especies forrajeras, por la alta nubosidad y baja temperatura; y 3) Época seca, de marzo a mayo, cuando la productividad de las plantas se reduce drásticamente por la escasez de lluvia (Hernández et al., 1990; Martínez et al., 2005).

En Isla, Veracruz, Martínez et al. (2008) reportaron la siguiente distribución estacional de B. dyctineura: 70, 13 y 8 $\%$, para las épocas de lluvias, nortes y seca, respectivamente. En el trópico húmedo de México, Cab et al. (2008) registraron 65,21 y $14 \%$ del rendimiento anual, en los pastos $B$. brizantha y $B$. dyctineura, para las épocas de lluvias, nortes y seca, respectivamente. Al evaluar tres frecuencias de corte (3, 5 y 7 semanas) en el pasto 'Mombaza' en el trópico seco de México, Ramírez et al. (2009) consignaron que la acumulación de forraje fue de 83 y 17 \% para las épocas de lluvias y de secas, respectivamente.

En los últimos años se han seleccionado varios genotipos de $B$. humidicola aptos para ser incorporados a los sistemas de producción animal. Sin embargo, antes de ser recomendados es necesario evaluar su potencial productivo mediante la determinación de sus características morfológicas. Al respecto, se ha indicado que las características como altura de planta, relación hoja:tallo, tasa de crecimiento, dinámica de ahijamiento (amacollamiento), expansión foliar, entre otras, presentan una relación directa con la productividad y calidad del forraje (Costa et al., 2009).

Dada la relevancia de contar con genotipos sobresalientes de esta especie en el trópico mexicano, es necesario estudiar sus patrones estacionales de crecimiento y su respuesta a los factores climáticos y de manejo. El objetivo de esta investigación fue evaluar el rendimiento total, composición morfológica y tasa de crecimiento de 23 genotipos de Brachiaria humidicola y de un híbrido de Brachiaria en las condiciones del trópico húmedo del Estado de Veracruz en México.

\section{MATERIALES Y MÉTODOS}

\section{Localización y descripción del sitio experimental}

El experimento se llevó a cabo en el Sitio Experimental "Papaloapan", ubicado en el km 66 de la carretera Cd. Alemán-Sayula, en el Municipio de Isla, Veracruz, a $18^{\circ}$ $06^{\prime} \mathrm{LN}$ y 953 $31^{\prime} \mathrm{LO}$, a una altitud de $65 \mathrm{~m}$. El clima de la zona es $\mathrm{Aw}_{0}$, que corresponde al más seco de los cálidos subhúmedos, con lluvias en verano y una precipitación promedio anual de $1000 \mathrm{~mm}$ de la cual $85 \%$ se distribuye de junio a noviembre, y con temperatura media anual de 25.7 ${ }^{\circ} \mathrm{C}$ (García, 1988). El suelo es acrisol órtico, franco arenoso, $\mathrm{pH}$ ácido (de 4 a 4.7), con bajos niveles de materia orgánica, nitrógeno, calcio, potasio, y niveles de medios a altos de fósforo y magnesio (Enríquez y Romero, 1999).

\section{Tratamientos y diseño experimental}

Se evaluaron 24 genotipos: 21 de $B$. humidicola procedentes del banco de germoplasma del Centro Internacional de Agricultura Tropical (CIAT) ubicado en Cali, Colombia, más tres variedades comerciales: CIAT $679 \mathrm{cv}$. 'Chetumal' y CIAT 6133 cv. 'Isleño' y el Hibrido 16 generado por el Instituto Nacional de Investigaciones Forestales Agrícolas y Pecuarias (Cuadro 1). Los 24 genotipos se sembraron con material vegetativo en 2005 , en parcelas de $4 \mathrm{~m}^{2}$. Una vez establecidos (tres meses después de la siembra), a las parcelas se les hizo un corte de uniformización a $8 \mathrm{~cm}$ de altura (en octubre) y posteriormente se mantuvieron bajo un régimen de cortes cada $35 \mathrm{~d}$ hasta el inicio del experimento.

Los 24 tratamientos se distribuyeron aleatoriamente en un diseño de bloques al azar con tres repeticiones, con los bloques ubicados en forma perpendicular a la pendiente del terreno que era de aproximadamente $10 \%$. El experimento se condujo de junio 2006 a mayo 2007, y abarcó las tres épocas del año (lluvia, nortes y seca), de acuerdo con la clasificación de estaciones del año propuestas por Hernández et al. (1990) y Martínez et al. (2005). Durante la época de lluvias los tratamientos se cosecharon cada $28 \mathrm{~d}$ (5 cortes) y en las épocas de nortes (2 cortes) y seca se cortaron cada 42 d (3 cortes). Debido a una infestación generalizada de Aeneolamia postica (mosca pinta o salivazo), en octubre se dio un corte a $5 \mathrm{~cm}$ para eliminar la plaga, por lo que la tasa de crecimiento de noviembre no se incluyó en resultados. 


\begin{tabular}{|c|c|}
\hline No & Número CIAT \\
\hline 1 & 6369 \\
\hline 2 & 16866 \\
\hline 3 & 16867 \\
\hline 4 & 16870 \\
\hline 5 & 16879 \\
\hline 6 & 16884 \\
\hline 7 & 16885 \\
\hline 8 & 16891 \\
\hline 9 & 16894 \\
\hline 10 & 26145 \\
\hline 11 & 26151 \\
\hline 12 & 26155 \\
\hline 13 & 26159 \\
\hline 14 & 26407 \\
\hline 15 & 26413 \\
\hline 16 & 26415 \\
\hline 17 & 26425 \\
\hline 18 & 26427 \\
\hline 19 & 26430 \\
\hline 20 & 679 (cv. 'Chetumal') \\
\hline 21 & 'Híbrido 16’ (INIFAP) \\
\hline 22 & 6133 (cv. 'Isleño') \\
\hline 23 & 6705 \\
\hline 24 & 26149 \\
\hline
\end{tabular}

\section{Variables medidas}

Rendimiento de forraje y composición morfológica. La producción de materia seca (MS), estacional y anual, se midió por parcela experimental y se transformó a rendimiento por hectárea $\left(\mathrm{kg} \mathrm{ha}^{-1}\right)$, mediante cortes efectuados cada $28 \mathrm{~d}$ durante la época de lluvias (26 de junio a 26 de octubre de 2005) y cortes cada $42 \mathrm{~d}$ en las épocas de nortes y seca (5 de diciembre 2005 al 1 de marzo de 2006, y del 10 de abril al 24 de mayo, respectivamente). En las dos frecuencias se cortó a $8 \mathrm{~cm}$ de altura sobre el nivel del suelo, todo el material vegetal incluido, en un marco fijo de $1 \mathrm{~m}^{2}$ que se colocó en el centro de cada parcela. El material fresco se pesó con una balanza granataria Ohaus $®$ (triple brazo) con capacidad de $610 \mathrm{~g}$; se obtuvo una muestra de aproximadamente $100 \mathrm{~g}$ en la cual se separaron los componentes: hojas, tallos, inflorescencias y material muerto. Las fracciones se secaron a $55^{\circ} \mathrm{C}$ por $48 \mathrm{~h}$ en una estufa de aire forzado Riossa ${ }^{\circledR}$ de 62 x 52 x $42 \mathrm{~cm}$, y se pesaron. Con los datos de peso fresco del total cosechado y el peso seco de los componentes morfológicos, se determinó el peso en gramos de materia seca, y se calculó el rendimiento en $\mathrm{kg} \mathrm{MS} \mathrm{ha-1.}$

Tasa de crecimiento. A los datos de peso seco registrados en cada corte se les aplicó la siguiente fórmula para calcular la tasa de crecimiento (Velasco et al., 2005):

$$
T C=\frac{F C}{T}
$$

Donde: $\mathrm{TC}=$ tasa de crecimiento $\left(\mathrm{kg} \mathrm{MS} \mathrm{ha} \mathrm{h}^{-1} \mathrm{~d}^{-1}\right)$; FC = forraje cosechado en cada corte $\left(\mathrm{kg} \mathrm{MS} \mathrm{ha}{ }^{-1}\right) ; \mathrm{T}=$ días transcurridos entre un corte y el siguiente.

Características climáticas durante el periodo experimental. La precipitación anual fue de $1542 \mathrm{~mm}$ y la temperatura promedio de $26^{\circ} \mathrm{C}$; la máxima temperatura ocurrió en el mes de mayo $\left(36.3^{\circ} \mathrm{C}\right.$ ) y la mínima en el mes de enero (16.1 $\left.{ }^{\circ} \mathrm{C}\right)$, como se ilustra en la Figura 1. La precipitación ocurrió en $74.8 \%$ durante la época de lluvias (junio a octubre), $23.7 \%$ en la época de nortes (noviembre a febrero), y $1.5 \%$ en la época seca (marzo a mayo).

\section{Análisis estadístico}

Se efectúo un análisis de varianza para cada variable, de acuerdo a un diseño de bloques completos al azar, y las medias se compararon con la prueba de Tukey (Steel y Torrie, 1988; SAS Institute, 2003). Los datos de rendimiento, tasa de crecimiento y composición morfológica, se agruparon por época del año y también se analizaron con el paquete estadístico de SAS (SAS Institute, 2003). El análisis se efectúo por intervalo entre cortes ( 28 y $42 \mathrm{~d}$ ), por época (lluvia, nortes y seca) y anual total (lluvias + nortes + seca). El modelo empleado fue:

$$
Y_{i j}=\mu+B_{i}+T_{j}+E_{i j}
$$

Donde: $Y_{i j}=$ variable de respuesta del i-ésimo ecotipo en el $\mathrm{j}$-ésimo bloque; $\mu=$ media general; $B i=$ efecto del i-ésimo bloque; $T_{j}=$ efecto del $\mathrm{j}$-ésimo tratamiento; $E_{i j}=$ error experimental.

\section{RESULTADOS Y DISCUSIÓN}

\section{Rendimiento de forraje}

El mayor y el menor rendimiento de forraje $(\mathrm{P} \leq 0.05)$ lo registraron dos variedades, el testigo cv. 'Chetumal' y el genotipo CIAT 26159, con 17353 y $11104 \mathrm{~kg}$ MS ha ${ }^{-1}$, respectivamente (Cuadro 2). Los mayores rendimientos ( $\mathrm{P}$ $\geq 0.05)$ se registraron en los testigos 'Chetumal' e 'Isleño', y en los genotipos CIAT 6705, 26425 y 26149 con 17 353, 16 222, 16213,15732 y $15093 \mathrm{~kg} \mathrm{MS} \mathrm{ha}^{-1}$, respectivamente. La distribución estacional del rendimiento de MS anual promedio de los 24 genotipos fue: 83,9 y $8 \%$ en las épocas de lluvias, nortes y seca, respectivamente.

En la estación de lluvias hubo diferencias $(\mathrm{P} \leq 0.05)$, entre genotipos en producción de forraje, donde el cv. 'Chetumal' y CIAT 26159 presentaron el máximo y mínimo rendimiento con 14046 y $8304 \mathrm{~kg} \mathrm{MS} \mathrm{ha}^{-1}$, respectivamente. En esta época la temperatura mínima promedio diaria fue de $22^{\circ} \mathrm{C}$ y la máxima de $33^{\circ} \mathrm{C}$, sin limitaciones de humedad 


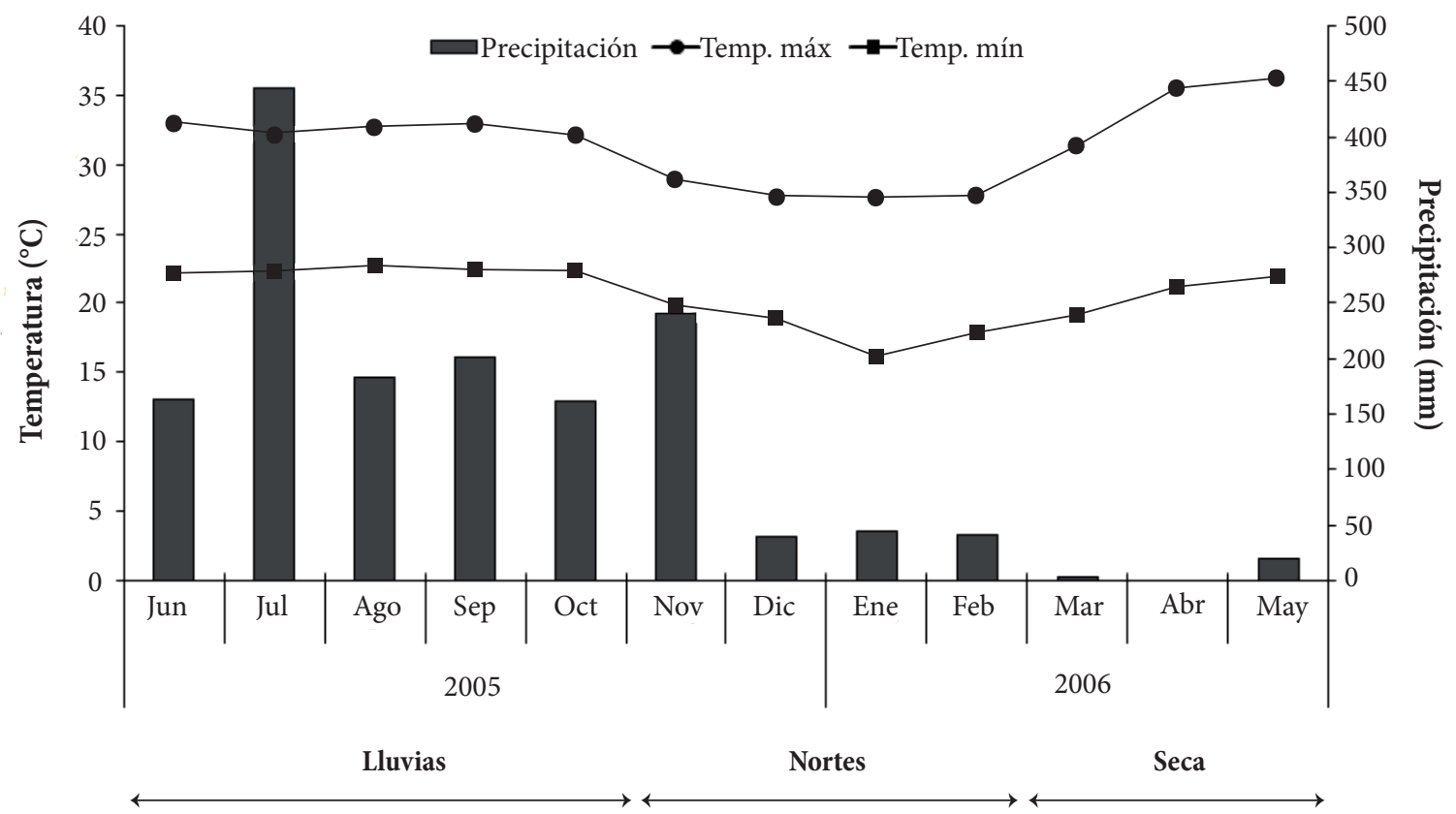

Figura 1. Características climáticas durante el periodo de estudio (junio 2005 a mayo 2006), en Isla, Veracruz.

Cuadro 2. Rendimiento anual y por época de materia seca $\left(\mathrm{kg} \mathrm{MS} \mathrm{ha}^{-1}\right)$, de genotipos de Brachiaria humidicola evaluados en Isla, Veracruz.

\begin{tabular}{|c|c|c|c|c|c|c|c|}
\hline \multirow[b]{2}{*}{ Genotipo } & \multicolumn{6}{|c|}{ Época } & \multirow[b]{2}{*}{ Total } \\
\hline & Lluvias & $\%$ & Nortes & $\%$ & Seca & $\%$ & \\
\hline $679+$ & $14046 \mathrm{a}$ & 81 & $1908 \mathrm{a}$ & 11 & 1399 abcdef & 8 & 17353 \\
\hline $6133++$ & $13529 \mathrm{ab}$ & 83 & 1366 cdefg & 8 & 1438 abcde & 9 & 16222 \\
\hline 6705 & $13529 \mathrm{abc}$ & 83 & 1327 cdefg & 8 & 1357 abcdef & 8 & 16213 \\
\hline 26425 & $12656 \mathrm{abcd}$ & 80 & 1444 bcdef & 9 & $1632 \mathrm{a}$ & 10 & 15732 \\
\hline 26149 & 11697 bcde & 78 & $1844 \mathrm{ab}$ & 12 & $1552 \mathrm{abc}$ & 10 & 15093 \\
\hline 26427 & 12146 abcde & 85 & 1174 defgh & 8 & 1021 abcdefgh & 7 & 14341 \\
\hline 26155 & 11584 bcde & 82 & 1154 efghi & 8 & 1307 abcdefg & 9 & 14046 \\
\hline 6369 & 11960 abcde & 87 & 963 ghijk & 7 & 855 defghi & 6 & 13778 \\
\hline 'Híbrido 16' & $11360 \mathrm{cde}$ & 83 & 1350 cdefg & 10 & 1032 abcdefgh & 8 & 13742 \\
\hline 26151 & $10432 \mathrm{efg}$ & 76 & $1592 \mathrm{abcd}$ & 12 & $1622 \mathrm{ab}$ & 12 & 13646 \\
\hline 16891 & 11791 bcde & 87 & 750 hijk & 6 & 977 cdefghi & 7 & 13518 \\
\hline 16885 & 11970 bcde & 89 & $707 \mathrm{jk}$ & 5 & 834 efghi & 6 & 13511 \\
\hline 26407 & 10926 defg & 81 & 1394 cdefg & 10 & 1191 abcdefgh & 9 & 13510 \\
\hline 16894 & 10613 defg & 79 & $1292 \mathrm{defg}$ & 10 & $1554 \mathrm{abc}$ & 12 & 13458 \\
\hline 16867 & 11996 abcde & 89 & $634 \mathrm{jk}$ & 5 & 816 fghi & 6 & 13446 \\
\hline 26413 & 10796 defg & 83 & 1044 fghij & 8 & 1237 abcdefgh & 9 & 13077 \\
\hline 26145 & $9458 \mathrm{fgh}$ & 75 & $1737 \mathrm{abc}$ & 14 & $1458 \mathrm{abcd}$ & 12 & 12653 \\
\hline 16866 & 11562 bcde & 92 & $596 \mathrm{k}$ & 5 & $477 \mathrm{i}$ & 4 & 12635 \\
\hline 26430 & $9153 \mathrm{gh}$ & 76 & $1579 \mathrm{abcd}$ & 13 & 1339 abcdef & 11 & 12071 \\
\hline 26415 & $10511 \mathrm{efg}$ & 88 & $681 \mathrm{jk}$ & 6 & 721 ghi & 6 & 11913 \\
\hline 16884 & $10202 \mathrm{efgh}$ & 88 & $728 \mathrm{ijk}$ & 6 & $696 \mathrm{hi}$ & 6 & 11625 \\
\hline 16879 & $10173 \mathrm{efgh}$ & 90 & $659 \mathrm{jk}$ & 6 & $534 \mathrm{i}$ & 5 & 11366 \\
\hline 16870 & $10214 \mathrm{efgh}$ & 90 & $598 \mathrm{k}$ & 5 & $548 \mathrm{i}$ & 5 & 11360 \\
\hline 26159 & $8304 \mathrm{~h}$ & 75 & 1494 abcde & 13 & 1305 abcdefg & 12 & 11104 \\
\hline Promedio & $11275 \mathrm{~A}$ & 83 & $1167 \mathrm{~B}$ & 9 & $1121 \mathrm{~B}$ & 8 & 13559 \\
\hline EEM & 176 & & 51 & & 47 & & 210 \\
\hline SIG & * & & * & & * & & * \\
\hline
\end{tabular}

Medias con letras minúsculas iguales en la misma columna no son estadísticamente diferentes, y medias con la letras mayúsculas iguales en la misma hilera no son estadísticamente diferentes (Tukey, 0.05); EEM = error estándar de la media; SIG = nivel de significancia; + 'Chetumal', ++'Isleño'; 5, 2 y 3 = cortes, para las épocas de lluvias, nortes y seca, respectivamente. 
(Figura 1), condiciones que favorecieron el crecimiento y permitieron el mayor rendimiento estacional de forraje. Algunos autores (McCluod y Bula, 1985; HernándezGaray et al., 2007; Festo et al., 2003; Sage y Kubein, 2007; Ramírez et al., 2009) consideran que el mayor crecimiento de las especies forrajeras ocurre entre 25 y $35^{\circ} \mathrm{C}$. Al tomar en cuenta que la temperatura también influye en la tasa de evaporación (Monteith, 1977), el crecimiento de las especies forrajeras en las regiones tropicales es mayor cuando la precipitación mensual excede en aproximadamente $150 \mathrm{~mm}$ a la tasa de evaporación (Enríquez et al., 1999). Por tanto, la interacción de la precipitación con la temperatura es un factor que afecta el crecimiento de los pastos (Martínez et al., 2008; Enríquez et al., 1999); además, hay una relación positiva entre la cantidad de lluvia y el rendimiento de materia seca (Martínez et al., 2008; Ramírez et al., 2009).

En la época de nortes la variación del rendimiento entre genotipos también fue significativa $(\mathrm{P} \leq 0.05)$. La mayor acumulaciónde forraje la registró el cv. 'Chetumal' con 1908 $\mathrm{kg} \mathrm{MS} \mathrm{ha-1,} \mathrm{y} \mathrm{los} \mathrm{menores} \mathrm{los} \mathrm{presentaron} \mathrm{los} \mathrm{genotipos}$ CIAT 16870 y 16866 , con 598 y $596 \mathrm{~kg} \mathrm{MS} \mathrm{ha}^{-1}$ (Cuadro 2). En esta época el rendimiento estuvo limitado por la incidencia de la plaga llamada "salivazo" al final de la época de lluvias (octubre 2006). La reducción de las horas luz y la alta nubosidad provocaron el descenso de la temperatura (Figura 1) y disminución de la radiación solar (Castillo et al., 2005; Enríquez et al., 1999), con temperaturas mensuales que oscilaron entre 18 a $28{ }^{\circ} \mathrm{C}$. Según Festo et al. (2003), las plantas forrajeras tropicales restringen su crecimiento hasta el grado de llegar a detenerlo, en respuesta a una temperatura inferior a $18{ }^{\circ} \mathrm{C}$. La cantidad de radiación fotosintéticamente activa incidente es afectada por la duración del día, la cual varía con las estaciones del año, latitud y altitud, y por otros factores ambientales de corta duración como la nubosidad (McKenzie et al., 1999).

En la época seca también hubo diferencias $(\mathrm{P} \leq 0.05)$ entre tratamientos, época en la que el genotipo CIAT 26425 con $1632 \mathrm{~kg} \mathrm{MS} \mathrm{ha}^{-1}$ registró el rendimiento más alto; en contraste, los genotipos de menor rendimiento fueron CIAT 16870, 16879 y 16866 con 548, 534 y $477 \mathrm{~kg} \mathrm{MS} \mathrm{ha}^{-1}$, respectivamente. Aunque la precipitación ocurrida en esta época sólo representó $1.5 \%$ del total anual (Figura 1), el rendimiento promedio fue similar al de la estación de nortes (Cuadro 2). Al respecto, Monteith (1997) consignó que el crecimiento vegetal puede continuar por un tiempo cuando deja de llover, como resultado del agua almacenada en el suelo.

En un estudio realizado en Isla, Veracruz con el cv. 'Isleño', en el que Martínez et al. (2008) evaluaron tres frecuencias y tres alturas de corte, los autores detectaron que independientemente de la frecuencia y altura de corte, la distribución estacional del rendimiento fue de 79,13 y $8 \%$ durante las épocas de lluvias, nortes y seca, con un rendimiento promedio anual de $11154 \mathrm{~kg} \mathrm{MS} \mathrm{ha-1}$ cuando se cortó a la mayor altura $(15 \mathrm{~cm})$. Los regímenes de corte que dieron el mayor rendimiento fueron los que se efectuaron cada cinco semanas en época de lluvias, nortes y seca $\left(9362,1538\right.$ y 1066 kg MS ha $\left.{ }^{-1}\right)$. Con excepción de la época de nortes, los rendimientos reportados por Martínez et al. (2008) fueron inferiores a los registrados en el presente estudio. En praderas de $B$. dictyoneura pastoreadas cada cinco semanas (en épocas de nortes y seca) y cada cuatro semanas (en épocas de lluvias) a una altura promedio de $12 \mathrm{~cm}$, Cab et al. (2007) registraron un rendimiento total de $15160 \mathrm{~kg} \mathrm{MS} \mathrm{ha}^{-1}$ con una distribución estacional de 20, 15 y $65 \%$ en las épocas de nortes, seca y lluvias, respectivamente. Esta distribución difiere de la del presente estudio, probablemente debido a la diferencia en la distribución de la precipitación durante el año, a las bajas temperaturas en la época de nortes y a la alta evapotranspiración en la época de sequía (Cab et al., 2008; Castillo et al., 2005).

En el mismo sitio y con los mismos genotipos utilizados en este experimento, Hernández-Garay et al. (2007) evaluaron el rendimiento de forraje durante las épocas de nortes y seca correspondientes al primer año de establecimiento, y registraron un rendimiento total promedio de 11556 kg MS ha ${ }^{-1}$, con una distribución estacional de $33 \%$ en la época de nortes (2005) y de $67 \%$ en la época de seca (2006); estos mismos autores señalaron que los genotipos más promisorios en rendimiento total fueron el 'Híbrido 16. CIAT 6133, CIAT 26245 y CIAT 26430, con 18 948, 16682,16093 y $15389 \mathrm{~kg} \mathrm{MS} \mathrm{ha}^{-1}$, respectivamente, y que la mayor producción de forraje en la época de nortes correspondió al Híbrido 16 mientras que durante la época seca correspondió al genotipo CIAT 26430. Estos resultados difieren de los registrados en este estudio para las mismas épocas, debido a que en el mes de mayo de 2006 llovió en abundancia (111 mm), lo que estimuló el crecimiento de todos los genotipos.

\section{Tasa de crecimiento}

La tasa de crecimiento (TC) tendió a descender en todos los genotipos conforme transcurrió cada época (Cuadro $3)$, pero con diferencias entre ellos $(\mathrm{P} \leq 0.05)$. Los valores máximos de TC se presentaron en el mes de junio con un promedio de $189 \mathrm{~kg} \mathrm{MS} \mathrm{ha}^{-1} \mathrm{~d}^{-1}$, para luego disminuir gradualmente hasta alcanzar su valor menor en el mes de mayo con $4 \mathrm{~kg} \mathrm{MS} \mathrm{ha}^{-1} \mathrm{~d}^{-1}$.

En la época de lluvias la TC promedio de los genotipos fue de 5.8 y 9.1 veces mayor que las registradas en la época de nortes y seca, respectivamente (Cuadro 4). Durante 
Cuadro 3. Tasa de crecimiento ( $\left.\mathrm{kg} \mathrm{MS} \mathrm{ha}^{-1} \mathrm{~d}^{-1}\right)$ mensual y promedio anual, de genotipos de Brachiaria humidicola evaluados en Isla, Veracruz.

\begin{tabular}{|c|c|c|c|c|c|c|c|c|c|c|}
\hline \multirow[b]{2}{*}{ Genotipo } & \multicolumn{6}{|c|}{2005} & \multicolumn{4}{|c|}{2006} \\
\hline & Junio & Julio & Agosto & Septiembre & Octubre & Diciembre & Enero & Marzo & Abril & Mayo \\
\hline $679+$ & $232 a$ & 82 bcd & $73 a b c$ & $61 \mathrm{a}$ & $54 \mathrm{c}$ & $22 \mathrm{ab}$ & $24 \mathrm{a}$ & $16 \mathrm{a}$ & $13 a b c$ & $4 \mathrm{bc}$ \\
\hline 6705 & $188 \mathrm{abc}$ & $124 \mathrm{a}$ & 39 efghi & $58 \mathrm{~b}$ & $75 a$ & $16 \mathrm{bcd}$ & $16 \mathrm{bc}$ & $13 \mathrm{ab}$ & $15 \mathrm{ab}$ & $4 \mathrm{bc}$ \\
\hline $6133++$ & $224 \mathrm{ab}$ & $102 a b c$ & $88 \mathrm{a}$ & 30 efg & $35 \mathrm{de}$ & $16 \mathrm{bcd}$ & $17 \mathrm{bc}$ & $11 \mathrm{abc}$ & $15 \mathrm{ab}$ & $8 \mathrm{a}$ \\
\hline 26425 & $235 a$ & $85 \mathrm{bcd}$ & 50 defgh & $39 \mathrm{def}$ & $43 \mathrm{~d}$ & $19 a b c$ & $10 \mathrm{fe}$ & $17 \mathrm{a}$ & $19 a$ & $3 c$ \\
\hline 26149 & $178 \mathrm{abc}$ & $125 \mathrm{a}$ & $41 \mathrm{ghi}$ & $41 \mathrm{cdef}$ & $46 \mathrm{~cd}$ & $25 \mathrm{a}$ & $19 \mathrm{ab}$ & $17 \mathrm{a}$ & $15 \mathrm{ab}$ & $5 \mathrm{bc}$ \\
\hline 26427 & $161 \mathrm{c}$ & $67 \mathrm{~cd}$ & $87 a$ & $50 \mathrm{bcæ}$ & $69 \mathrm{ab}$ & $16 \mathrm{bcd}$ & $12 \mathrm{de}$ & $10 \mathrm{bc}$ & $11 \mathrm{bcd}$ & $3 c$ \\
\hline 26155 & $199 \mathrm{abc}$ & $81 \mathrm{bcd}$ & 54 cdefg & $40 \mathrm{def}$ & $39 \mathrm{~d}$ & $14 \mathrm{cde}$ & 14 cde & $12 \mathrm{ab}$ & $12 \mathrm{bcd}$ & $7 \mathrm{a}$ \\
\hline 6369 & $205 a b c$ & $108 \mathrm{ab}$ & 37 fghi & 39 def & $38 \mathrm{de}$ & $12 \mathrm{def}$ & $11 \mathrm{de}$ & $8 \mathrm{c}$ & 8 cde & $5 \mathrm{bc}$ \\
\hline 16885 & $221 \mathrm{ab}$ & $88 \mathrm{abcd}$ & 54 cdefg & 38 def & $26 \mathrm{ef}$ & $10 \mathrm{ef}$ & $8 \mathrm{f}$ & $9 c$ & $8 \mathrm{cde}$ & $3 c$ \\
\hline 16867 & $229 \mathrm{ab}$ & $72 \mathrm{bcd}$ & $57 \mathrm{cde}$ & $38 \mathrm{def}$ & $32 \mathrm{e}$ & $8 \mathrm{fg}$ & $7 \mathrm{f}$ & $8 \mathrm{c}$ & 9 cde & $3 c$ \\
\hline 'Híbrido 16' & $153 c$ & $80 \mathrm{bcd}$ & $80 \mathrm{ab}$ & $47 \mathrm{bc}$ & $44 \mathrm{~d}$ & $18 \mathrm{abc}$ & 14 cde & $10 \mathrm{bc}$ & $10 \mathrm{bcd}$ & $4 \mathrm{bc}$ \\
\hline 16891 & $202 a b c$ & $77 \mathrm{bcd}$ & $63 \mathrm{abcd}$ & $35 \mathrm{def}$ & $43 \mathrm{~d}$ & $10 \mathrm{ef}$ & $8 \mathrm{f}$ & $9 \mathrm{c}$ & $10 \mathrm{bcd}$ & $4 \mathrm{bc}$ \\
\hline 26407 & $190 \mathrm{abc}$ & $82 \mathrm{bcd}$ & 39 efghi & 45 ced & $35 \mathrm{de}$ & $18 a b c$ & 15 cde & $13 \mathrm{ab}$ & $13 \mathrm{abc}$ & $3 c$ \\
\hline 26151 & $187 \mathrm{abc}$ & $68 \mathrm{bcd}$ & $28 \mathrm{ghi}$ & $25 \mathrm{fg}$ & $64 \mathrm{bc}$ & $21 \mathrm{ab}$ & $17 \mathrm{bc}$ & $17 \mathrm{a}$ & $14 \mathrm{ab}$ & $8 \mathrm{a}$ \\
\hline 16894 & $178 \mathrm{abc}$ & $71 \mathrm{bcd}$ & 56 bcde & 30 efg & $43 \mathrm{~d}$ & $16 \mathrm{bcd}$ & $15 \mathrm{bcd}$ & $13 \mathrm{ab}$ & $18 \mathrm{a}$ & $7 a$ \\
\hline 26413 & $154 \mathrm{c}$ & $101 \mathrm{abc}$ & 42 efghi & $42 \mathrm{cde}$ & $47 \mathrm{~cd}$ & $12 \mathrm{def}$ & $13 \mathrm{cde}$ & $11 \mathrm{abc}$ & $14 \mathrm{ab}$ & $5 \mathrm{bc}$ \\
\hline 16866 & $209 a b c$ & $81 \mathrm{bcd}$ & $67 \mathrm{abc}$ & $39 \mathrm{def}$ & $17 \mathrm{~g}$ & $8 \mathrm{fg}$ & $7 \mathrm{f}$ & $4 \mathrm{e}$ & $5 \mathrm{e}$ & $2 \mathrm{~d}$ \\
\hline 26145 & $161 \mathrm{c}$ & $65 \mathrm{~cd}$ & $20 \mathrm{i}$ & $36 \mathrm{def}$ & $56 \mathrm{c}$ & $21 \mathrm{ab}$ & $20 \mathrm{ab}$ & $16 \mathrm{a}$ & $14 \mathrm{ab}$ & $5 \mathrm{bc}$ \\
\hline 26415 & $189 \mathrm{abc}$ & $71 \mathrm{bcd}$ & 38 fghi & $47 \mathrm{bcd}$ & $30 \mathrm{e}$ & $6 \mathrm{~h}$ & $12 \mathrm{de}$ & $7 \mathrm{~cd}$ & $8 \mathrm{cde}$ & $2 \mathrm{~d}$ \\
\hline 16884 & $176 \mathrm{abc}$ & $68 \mathrm{bcd}$ & 52 cdefg & 45 bcde & $23 \mathrm{f}$ & $8 \mathrm{fg}$ & $9 \mathrm{ef}$ & $8 \mathrm{c}$ & $6 \mathrm{de}$ & $3 c$ \\
\hline 26430 & $152 \mathrm{c}$ & $78 \mathrm{ncd}$ & 26 ghi & $29 \mathrm{efg}$ & $41 \mathrm{~d}$ & $20 \mathrm{ab}$ & $18 \mathrm{ab}$ & $13 \mathrm{ab}$ & $14 \mathrm{ab}$ & $5 \mathrm{bc}$ \\
\hline 16870 & $172 b c$ & $98 \mathrm{abc}$ & 43 efghi & $34 \mathrm{def}$ & $17 \mathrm{~g}$ & $6 \mathrm{~h}$ & $8 \mathrm{f}$ & $5 \mathrm{~d}$ & $6 \mathrm{de}$ & $2 \mathrm{~d}$ \\
\hline 16879 & $182 \mathrm{abc}$ & $66 \mathrm{~cd}$ & 52 cdefg & 30 efg & $32 \mathrm{e}$ & $6 \mathrm{~h}$ & $10 \mathrm{ef}$ & $6 \mathrm{~d}$ & $5 \mathrm{e}$ & $2 \mathrm{~d}$ \\
\hline 26159 & $161 \mathrm{c}$ & $51 d$ & $21 \mathrm{hi}$ & $17 \mathrm{~g}$ & $46 \mathrm{~cd}$ & $21 \mathrm{ab}$ & 14 cde & $14 \mathrm{ab}$ & $13 \mathrm{a}$ & $4 \mathrm{bc}$ \\
\hline Promedio & $189 \mathrm{~A}$ & 83 B & $50 \mathrm{C}$ & $39 \mathrm{C}$ & $42 \mathrm{C}$ & $14 \mathrm{D}$ & 13D & 11D & $12 \mathrm{D}$ & $4 \mathrm{E}$ \\
\hline EEM & 3.5 & 2.1 & 2.4 & 1.3 & 1.9 & 0.7 & 0.7 & 0.5 & 0.5 & 0.2 \\
\hline SIG & * & * & * & * & * & * & * & * & * & * \\
\hline
\end{tabular}

Medias con letras minúsculas iguales en la misma columna no son estadísticamente diferentes, y medias con la letras mayúsculas iguales en la misma hilera no son estadísticamente diferentes (Tukey, 0.05). EEM = error estándar de la media; SIG = nivel de significancia; + = 'Chetumal', ++ = 'Isleño'; 5, 2 y 3 = cortes para las épocas de lluvias, nortes y seca, respectivamente.

el periodo de lluvias el cv. 'Chetumal' presentó la mayor TC (100 kg MS ha- $\left.\mathrm{d}^{-1}\right)$, mientras que CIAT 26159 tuvo la menor con $59 \mathrm{~kg} \mathrm{MS} \mathrm{ha}^{-1} \mathrm{~d}^{-1}(\mathrm{P} \leq 0.05)$. También hubo diferencias entre genotipos en las épocas de nortes y seca $(\mathrm{P}$ $\leq 0.05)$, donde el cv. 'Chetumal' (23 kg MS ha $\left.{ }^{-1} \mathrm{~d}^{-1}\right)$ y CIAT 26425 y 26151 (13 kg MS ha- $\left.\mathrm{kg}^{-1}\right)$ registraron las mayores tasas. Las diferencias en TC entre épocas se atribuyen a diferencias climáticas y a diferencias de manejo del pastizal durante la fase experimental, ya que estos factores afectan la productividad de la planta, especialmente a la actividad fotosintética (Hodgson et al., 1999).

Al evaluar 16 genotipos de Brachiaria en Isla, Veracruz, Enríquez y Romero (1999) obtuvieron tasas promedio de $72 \mathrm{~kg} \mathrm{MS} \mathrm{ha}^{-1} \mathrm{~d}^{-1}$ en la época de lluvias, de $7 \mathrm{~kg} \mathrm{MS} \mathrm{ha}^{-1}$ $\mathrm{d}^{-1}$ en la de nortes y de $27 \mathrm{~kg} \mathrm{MS} \mathrm{ha}^{-1} \mathrm{~d}^{-1}$ en la época seca, valores que son diferentes a los reportados en el presente estudio. Por su parte, Martínez et al. (2008) registraron tasas promedio de 58, 12 y $9 \mathrm{~kg} \mathrm{MS} \mathrm{ha}^{-1} \mathrm{~d}^{-1}$ para las épocas de lluvias, nortes y seca, en el genotipo CIAT 6133 cosechado a tres frecuencias y tres alturas de corte; en las tres épocas la mayor TC se presentó en la mayor altura de corte porque quedó más tejido foliar remanente. En otro estudio efectuado en el mismo sitio durante las épocas de nortes y seca, Hernández-Garay et al. ( 2007) reportaron tasas promedio de 42 y $65 \mathrm{~kg} \mathrm{MS} \mathrm{ha}^{-1} \mathrm{~d}^{-1}$, valores muy superiores a los aquí encontrados, lo cual pudiera atribuirse a que en el presente periodo de evaluación las condiciones climáticas fueron atípicas, particularmente en la época seca cuando llovió $111 \mathrm{~mm}$ a principios de mayo. Cab et al. (2008) compararon la TC de $B$. dictyoneura en unicultivo $v$ s. la asociación $B$. dictyneura-Arachis pintoi, durante las tres épocas del año (lluvias, nortes y seca); observaron que la pradera de $B$. dictyoneura tuvo una TC 50 \% mayor que la asociación, en las tres estaciones.

\section{Composición morfológica}

La composición morfológica varió $(\mathrm{P} \leq 0.05)$ entre genotipos dentro de cada época, y también entre épocas del año (Figura 2). Las hojas fueron los organos que más contribuyeron al rendimiento en todas las épocas, 
Cuadro 4. Tasa de crecimiento estacional ( $k g$ MS ha-1 $\left.\mathrm{d}^{-1}\right)$ de genotipos de Brachiaria humidicola evaluados en Isla, Veracruz.

\begin{tabular}{|c|c|c|c|}
\hline \multirow[b]{2}{*}{ Genotipo } & \multicolumn{3}{|c|}{ Época } \\
\hline & Lluvias & Nortes & Seca \\
\hline $679+$ & $100 \mathrm{a}$ & $23 \mathrm{a}$ & 11 abcde \\
\hline 6705 & $97 \mathrm{ab}$ & $16 \mathrm{cdefg}$ & 11 abcde \\
\hline $6133++$ & $96 \mathrm{abc}$ & $16 \mathrm{cdefg}$ & 11 abcde \\
\hline 26425 & $90 \mathrm{abcd}$ & 14 bcdef & $13 \mathrm{a}$ \\
\hline 26149 & 86 bcde & $22 \mathrm{ab}$ & $12 \mathrm{ab}$ \\
\hline 26427 & 87 abcde & 14 defg & 8 bcdefg \\
\hline 26155 & 83 bcde & 14 defgh & 10 abcdef \\
\hline 6369 & 85 abcde & 11 ghijk & 7 defgh \\
\hline 16885 & 85 abcde & $9 \mathrm{ijk}$ & 7 defgh \\
\hline 16867 & 86 abcde & $8 \mathrm{jk}$ & $6 \mathrm{fgh}$ \\
\hline 'Híbrido 16' & $81 \mathrm{cdef}$ & $16 \mathrm{cdefg}$ & 8 bcdefg \\
\hline 16891 & 84 bcde & $9 \mathrm{ijk}$ & 8 bcdefg \\
\hline 26407 & 78 defg & 17 bcdefg & 9 abcdefg \\
\hline 26151 & 75 efg & $19 \mathrm{abcd}$ & $13 \mathrm{a}$ \\
\hline 16894 & 76 defg & $15 \mathrm{cdefg}$ & $12 \mathrm{ab}$ \\
\hline 26413 & 77 defg & 12 fghij & 10 abcdef \\
\hline 16866 & 83 bcde & $7 \mathrm{k}$ & $4 \mathrm{~h}$ \\
\hline 26145 & $68 \mathrm{fgh}$ & $21 \mathrm{abc}$ & $12 \mathrm{ab}$ \\
\hline 26415 & 75 defg & $9 \mathrm{jk}$ & $6 \mathrm{fgh}$ \\
\hline 16884 & 73 efgh & $9 \mathrm{ijk}$ & $6 \mathrm{fgh}$ \\
\hline 26430 & $65 \mathrm{gh}$ & 19 abcde & 11 abcde \\
\hline 16870 & 73 efgh & $7 \mathrm{k}$ & $4 \mathrm{~h}$ \\
\hline 16879 & 73 efgh & $8 \mathrm{jk}$ & $4 \mathrm{~h}$ \\
\hline 26159 & $59 \mathrm{~h}$ & 18 abcde & 10 abcdef \\
\hline Promedio & $81 \mathrm{~A}$ & $14 \mathrm{~B}$ & $9 \mathrm{C}$ \\
\hline EEM & 1.3 & 0.6 & 0.4 \\
\hline SIG & * & $*$ & * \\
\hline
\end{tabular}

Medias con letras minúsculas iguales en la misma columna no son estadísticamente diferentes, y medias con letras mayúsculas iguales en la misma hilera no son estadísticamente diferentes (Tukey, 0.05). EEM = error estándar de la media; SIG $=$ nivel de significancia; $+=$ 'Chetumal'; ++ = 'Isleño'; 5, 2 y $3=$ cortes para las épocas de lluvias, nortes y seca, respectivamente.

y la aportación en las épocas varió en el siguiente orden descendente $(\mathrm{P} \leq 0.05)$ : nortes $>$ seca $>$ lluvias, con promedios de $77.6,71.7$ y $57.6 \%$, respectivamente. Similares resultados fueron reportados por Martínez et al. (2008), quienes registraron la mayor proporción de hojas en la época de lluvias, lo que atribuyeron a la mayor tasa de recambio de tejido, como reportaron Ramírez et al. (2010). La mayor contribución de los tallos se registró en la época de lluvias (28.9\%), en tanto que el material muerto (MM) fue más abundante en la época seca (21.1\%). La escasa contribución de tallos y MM al rendimiento durante la época de nortes pudo deberse a la baja temperatura registrada, mientras que en la época seca el aumento de MM puede atribuirse a que la escasez de agua incrementó la senescencia de tejidos (Ramírez et al., 2009).

En la época de lluvias la proporción de hojas varió de 46 a $70 \%$, valores que corresponden al genotipo CIAT 6705 y al 'Híbrido 16' (Figura 2). La proporción relativa de tallos en esta época fluctúo de 17 a $39 \%$, y el genotipo CIAT 6705 superó $(\mathrm{P} \leq 0.05)$ al resto. El porcentaje de material muerto varió entre genotipos $(\mathrm{P} \leq 0.05)$, donde CIAT 16879 registró el valor mayor (22\%). Esta época fue la que más afectó el comportamiento de las características morfológicas y es cuando hubo la mayor contribución de tallos y de $\mathrm{MM}$ al rendimiento, atribuible a la mayor tasa de generación de estos tejidos, como ocurre en especies tropicales (Ramírez et al., 2010). Al evaluar el efecto del pastoreo cada $28 \mathrm{~d} v s$. 100 y $95 \%$ de intercepción luminosa (IL) en B. brizantha, Pedreira et al. (2009) obtuvieron mayores rendimientos de forraje con cortes cada 28 d y $100 \%$ IL, pero recomendaron cosechar las praderas cuando intercepten $95 \%$ de la radiación que es cuando aumenta el porcentaje de tallos y MM, particularmente durante el verano (época de lluvias).

En la época de nortes también hubo diferencias $(\mathrm{P} \leq 0.05)$ entre genotipos en cuanto a la contribución de componentes del rendimiento, aunque los valores fueron menores a los registrados en la época de lluvias. En hojas la diferencia entre genotipos fue de $17 \%$, donde el menor valor fue para CIAT $16884(66 \%)$ y el mayor para CIAT 16891 y CIAT 26407 (ambos con $86 \%$ ). La aportación de tallos fue diferente entre los genotipos CIAT 26151 y 16867 , con valores de 21 y $3 \%$; el resto de genotipos tuvieron valores intermedios ( $11 \%$, en promedio). En material muerto, los genotipos con mayor contribución $(\mathrm{P} \leq 0.05)$ fueron CIAT 16886 y CIAT 16870 (21\%), y los menores fueron CIAT 16891 y CIAT 26413 (6\%), mientras que en el resto no hubo diferencias (promedio de $11 \%$ ). El alto porcentaje de hojas y la escasa presencia de tallos se debe a que en especies tropicales la elongación del tallo resulta inhibida por las bajas temperaturas (Martínez et al., 2008), como las que aquí se registraron en la época de nortes (Figura 1).

En la época seca el 'Híbrido 16’ y CIAT 16879 tuvieron la mayor aportación de MM (30 \%), y CIAT $26155 \mathrm{y} c v$. 'Isleño' presentaron los menores ( 11 y $12 \%$, respectivamente). En este periodo los tallos contribuyeron menos $(\mathrm{P} \leq 0.05)$ al rendimiento, con valores de 2 y $14 \%$ para el 'Híbrido 16 ' y CIAT 26151, respectivamente; en el resto de los genotipos no hubo diferencias, con un promedio de $7 \%$. El porcentaje de hojas varió de 60 a $82 \%$; los genotipos CIAT 6705 y CIAT 26155 presentaron el mayor y menor valor $(\mathrm{P} \leq 0.05)$, respectivamente. Durante este periodo hubo la menor tasa de regeneración de tejido, como también reportaron Ramírez et al. (2010), debido al estrés hídrico (Figura 1); en cambio, eso favoreció al MM (Figura 2).

Los cambios observados en la composición morfológica respondieron a la interacción de los genotipos con las condiciones climáticas ocurridas en el transcurso del estudio. 

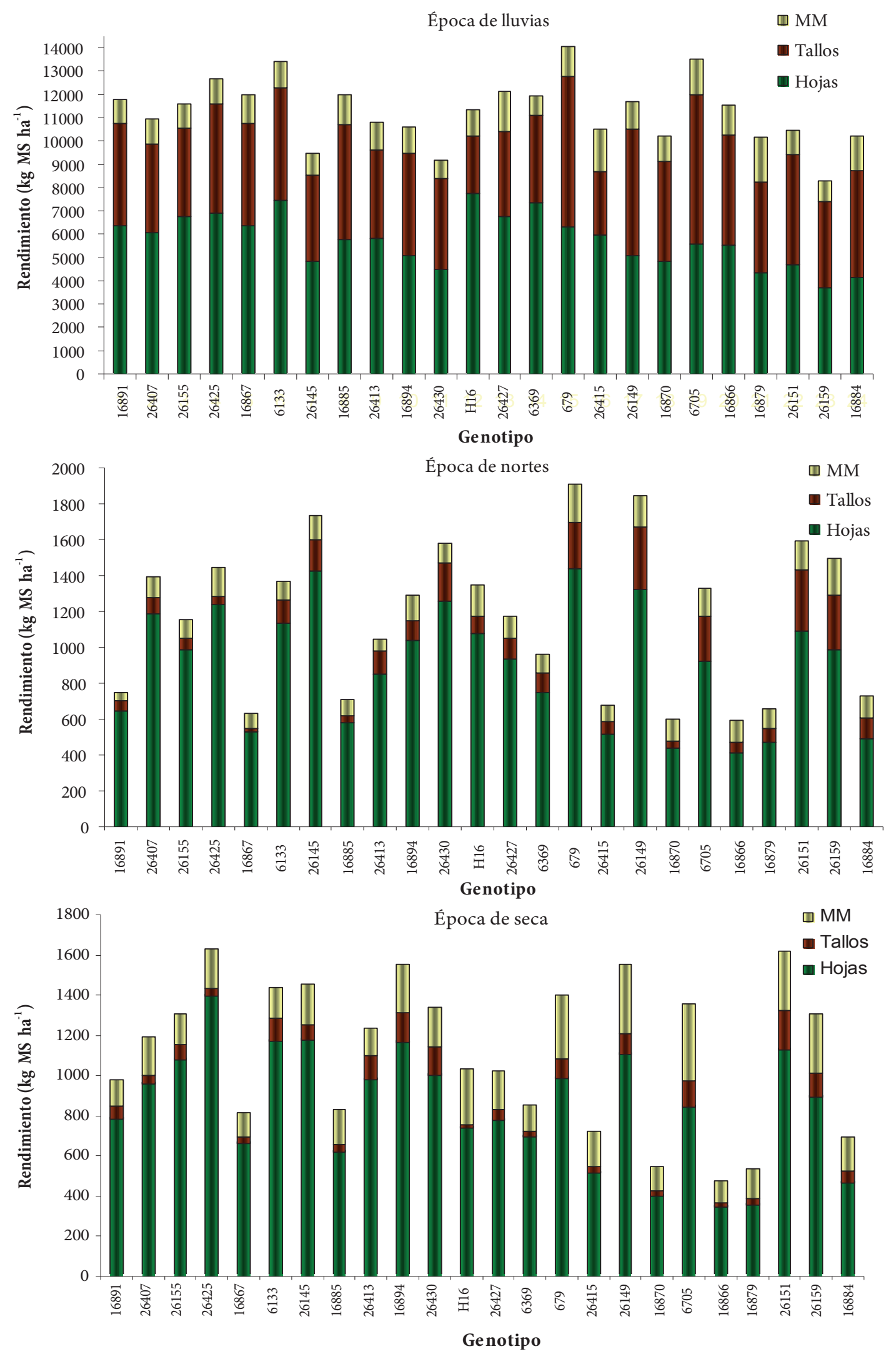

Figura 2. Composición morfológica y su variación estacional del rendimiento (en $\mathrm{kg} \mathrm{MS} \mathrm{ha}^{-1}$ ), de genotipos de Brachiaria humidicola evaluados en Isla, Veracruz. 
Durante la época de lluvias, con mayor temperatura y disponibilidad de humedad, hubo un crecimiento acelerado de las partes vegetales y ello se reflejó en abundante producción de tallos. Esto concuerda con lo descrito por McCluod y Bula (1985) quienes mencionaron que la temperatura óptima para el crecimiento de tallos de gramíneas tropicales oscila entre 30 y $35^{\circ} \mathrm{C}$, que se asocia con una disminución en la proporción de hojas cuyo crecimiento óptimo ocurre entre 20 y $25^{\circ} \mathrm{C}$ (Robson et al., 1988).

\section{CONCLUSIONES}

Los genotipos que registraron los mayores rendimientos anuales de forraje fueron el $c v$. 'Chetumal' y CIAT 6133 y CIAT 6705. La distribución promedio a través de estaciones fue de 83,9 y $8 \%$ para las épocas de lluvias, nortes y seca, respectivamente. En la época de lluvias los genotipos cv. 'Chetumal' y CIAT 26159 presentaron los mayores rendimientos. En la época de nortes el $c v$. 'Chetumal' fue el más tolerante al frío. El genotipo CIAT 26425, con $1632 \mathrm{~kg}$ MS $\mathrm{ha}^{-1}$, fue el más tolerante a sequía. Las hojas fueron el componente morfológico que más contribuyó al rendimiento de todos los genotipos, en las tres épocas del año.

\section{BIBLIOGRAFÍA}

Argel P J (2005) Contribución de los forrajes mejorados a la productividad ganadera en sistemas de doble propósito. In: XIX Reunión de la Asociación Latinoamericana de Producción Animal. Tampico, Tamaulipas, México. pp:42-50.

Cab J F E, Q J F Enríquez, P J Pérez, G A Hernández, H J G Herrera, J E Ortega, C A R Quero (2008) Potencial productivo de tres especies de Brachiaria en monocultivo y asociadas con Arachis pintoi en Isla, Veracruz. Téc. Pecu. Méx. 46:317-332.

Castillo G E, B Valles de la Mora, L Mannettje, S A Aluja (2005) Efecto de introducir Arachis pintoi sobre variables del suelo de pasturas de gramas nativa del trópico húmedo mexicano. Téc. Pecu. Méx. 43:287-295.

Costa N L, V T Paulino, C R Townsend, J A Magalhães, J R C Oliveira (2007) Desempenho agronómico de genotipos de Brachiaria brizantha em diferentes idades de corte em Porto Velho, Rondônia, Brasil. Rev Electron Vet 8:1-5. Disponible en: http://www.veterinaria.org/ revistas/redvet/n080807/080704.pdf. (Mayo, 2009).

Cruz P, M Boval (2000) Effect of nitrogen on some morphogenetic traits of temperate and tropical perennial forage grasses. In: Grassland Ecophysiology and Grazing Ecology. G Lemaire, J Hogson, A de Moraes, C Nabinger, P C de F Carvalho (eds). CAB International, Wallingford Oxon OX10 8DE, UK. pp:151-168.

Enríquez Q J F, N F Meléndez, A E D Bolaños (1999) Tecnología para la Producción y Manejo de Forrajes Tropicales en México. INIFAP CIRGOC. Campo Experimental Papaloapan. Libro Técnico No. 7. Veracruz México. $262 \mathrm{p}$.

Enríquez Q J F, M Romero (1999) Tasa de crecimiento estacional a diferentes edades de rebrote de 16 ecotipos de Brachiaria spp. en Isla, Veracruz. Agrociencia 33:141-148.
FAO (2006) http://apps.fao.org/PAGE/COLLECTIONS/SUBSET/AGRICULTURE. (Marzo, 2010).

Festo J M, N A Sabed, A R Jeremy (2003) The impact of temperature on leaf appearance in bamba groundnut landraces. Crop Sci. 43:13751379.

García E (1988) Modificaciones al Sistema de Clasificación Climática de Köppen. $4^{\text {a }}$ ed. Universidad Nacional Autónoma de México. México, D. F. 217 p.

Hernández T, B Valles, E Castillo (1990) Evaluación de gramíneas y leguminosas forrajeras en Veracruz, México. Pasturas Trop. 12:29-33.

Hodgson J, N P Matthews, C Matthew, J R Lucas (1999) Pasture measurements. In: New Zealand Pasture and Crop Science. J White, J Hodgson (eds). Auckland, N.Z. Oxford University. pp:59-65

Keller-Grein G, L B Maass, J Hanson (1996) Natural variation in Brachiaria and existing germoplasm collections. In: Brachiaria: Biology, Agronomy and Improvement. J W Miles, B L Maass, C B do Valle (eds). CIAT and Embrapa. CIAT publication 259. Cali, Colombia. pp:6-42.

Ludlow M M (1980) Stress physiology of tropical pasture plants. Trop. Grassland 12:136-145.

Martínez M D, A Hernández-Garay, Q J F Enríquez, P J Pérez, M S S González, H J G Herrera (2008) Producción de forraje y componentes del rendimiento de pasto Brachiaria humidicola CIAT 6133 con diferente manejo de defoliación. Téc. Pecu. Méx. 46:427-438.

McCloud D E, R J Bula (1985) Climatic factors in forage production. In: The Science of Grassland Agricultural. M E Heat, R F Barnes, B Metcalfe (eds). Iowa State University. Iowa, U.S.A. pp:33-42.

McKenzie B A, P D Kemp, D J Moot, C Matthew, R L Lucas (1999) Environmental effects on plant growth and development. In: New Zealand Pastures and Crop Science. J White, J Hodgson (eds). Oxford University Press. pp:29-44.

Monteith L J (1997) Climate. In: Ecophysiology of Tropical Crops. P T Alvim T T Kozlowski (eds). Academic Press, New Cork, U.S.A pp:1-28.

Pedreira B C, C G S Pedreira, S C Da Silva (2009) Acumulo de forragem durante a rebrotacao de campim-xaraes submetido a tres estrategias de desfolhação. Rev. Brãs. Zoot. 34:618-625.

Ramírez R O, A Hernández-Garay, C S Da Silva, P J Pérez, Q J F Enríquez, C A R Quero, H J G Herrera, N A Cervantes (2009) Acumulación de forraje, crecimiento y características estructurales del pasto Mombaza (Panicum maximun Jacq) cosechado a diferentes intervalos de corte. Téc. Pecu. Méx. 47:203-213.

Ramírez R 0, A Hernández-Garay, C S Da Silva, P J Pérez, S J Souza jr, R R Castro, Q J F Enríquez (2010) Características morfogenéticas y su influencia en el rendimiento del pasto Mombaza, cosechado a diferentes intervalos de corte. Trop. Subtrp. Agroecosys. 12:303-311.

Robson M J, G J A Ryle, J Woledg (1988) The grass crop. In: The Physiological Basis of Production. M B Jones, A Lazenby (eds). Chapman and Hall Ltd. London, Great Britain. pp:37-38.

Sage F R, S D Kubein (2007) The temperature response of C3 and C4 photosynthesis. Plant Cell Environ. 30:1086-1106.

SAS Instuitute (2003) SAS language: Release 8.2 for Windows. SAS Institute Inc. Cary, NC, USA. 956 p.

Steel R G D, J H Torrie (1988) Bioestadística, Principios y Procedimientos. 2a ed. Mc Graw Hill. pp:368-386.

Velasco Z M E, A Hernández-Garay, H V A González (2005) Rendimiento y valor nutritivo del ballico perenne (Lolium perenne L.) en respuesta a la frecuencia de corte. Téc. Pecu. Méx. 43:247-258. 